Des scènes chantées et du Film d'Art, de l'accompagnement musical approprié et du film musical : deux ou trois notes sur une histoire de la musique du cinéma muet

\title{
Édouard Arnoldy
}

\section{(2) OpenEdition \\ 1 Journals}

\section{Édition électronique}

URL : https://journals.openedition.org/1895/243

DOI : 10.4000/1895.243

ISBN : 978-2-8218-1026-6

ISSN : $1960-6176$

\section{Éditeur}

Association française de recherche sur l'histoire du cinéma (AFRHC)

\section{Édition imprimée}

Date de publication : 1 octobre 2002

ISBN : 2-913758-35-5

ISSN : 0769-0959

Référence électronique

Édouard Arnoldy, «Des scènes chantées et du Film d'Art, de l'accompagnement musical approprié et du film musical : deux ou trois notes sur une histoire de la musique du cinéma muet », 1895. Mille huit cent quatre-vingt-quinze [En ligne], 38 | 2002, mis en ligne le 08 février 2007, consulté le 17 avril 2022. URL : http://journals.openedition.org/1895/243 ; DOI : https://doi.org/10.4000/1895.243

Ce document a été généré automatiquement le 17 avril 2022.

(c) AFRHC 


\title{
Des scènes chantées et du Film d'Art, de l'accompagnement musical approprié et du film musical : deux ou trois notes sur une histoire de la musique du cinéma muet
}

\author{
Édouard Arnoldy
}

1 Cet article a pour ambition non pas de proposer une histoire de la musique des films muets, une histoire monolithique et autosuffisante, mais bien d'entrecroiser certains développements des histoires du cinéma, de l'accompagnement musical du spectacle cinématographique et de diverses pratiques culturelles et médiatiques (le phonographe, le téléphone, le café-concert, le théâtre, l'Opéra). Avec pour souci de dénouer quelques-uns des fils entre lesquels s'enchevêtre une histoire générale de la musique de cinéma, un temps d'arrêt sur la synchronisation mécanique de l'image et des sons mérite sans doute d'être conduit. Dans la foulée, un égard certain va être accordé aux «films chantants et parlants" des années dix, une époque où, conjointement, il est souvent débattu de l'opportunité d'accompagner les films de musique (et/ou de bruitages) et de consacrer un Art cinématographique ${ }^{1}$. Enfin, un peu d'attention sera octroyée à la fin des années vingt et à la place un peu particulière qu'occupe la musique dans le mouvement inhérent au passage du muet au parlant.

2 Ainsi, en janvier et juillet 1908, Gaumont met sous presse ses Catalogues pour Projections Parlantes, des publications singulières en ce qu'elles ne laissent planer aucun doute sur la place particulière accordée là au «film chantant et parlant ». Alors qu'en 1908 Pathé songe surtout à organiser l'industrie naissante du cinéma, Gaumont - dont les recherches sur le son et la couleur sont notoires - pense peut-être davantage l'avenir en termes d'inventions, d'innovations ou de perfectionnements techniques. Dans ces volumes, seules quelques lignes sont consacrées aux sujets des films, très peu aux éventuels choix de "mise en scène» ou de répertoire. En plus de la performance 
technique de son matériel, en ces pages mise entre parenthèses, la société Gaumont n'en garantit pas moins la qualité de ses films. D'un opportunisme certain, la compagnie - dont on connaît les efforts de persuasion quand il est question pour elle de promouvoir ses appareils - clame haut et fort son souci de ne jamais négliger les « effets artistiques » de ses vues :

Nous ne reculons devant aucun sacrifice pour donner à nos phono-scènes le maximum d'effet artistique qu'il soit possible d'obtenir tant par le choix des décors, l'emplacement de la prise de vue et l'interprétation de l'idée de l'auteur du scénario. Si vous consultez nos listes de phono-scènes, vous pourrez remarquer que nous en avons déjà édité plusieurs centaines, notre répertoire s'enrichit de plus en plus de sujets toujours plus artistiques. ${ }^{2}$

Gages supplémentaires d'un succès énorme, les "sujets toujours plus artistiques » c'est-à-dire des films dont les décors, le scénario et la prise de vue bénéficient de soins particuliers - sont d'entrée mis à l'avant plan. Quand elle insiste sur les "effets artistiques " de ces vues, la compagnie Gaumont n'est sans doute pas insensible aux sirènes qui annoncent à tout rompre l'avènement du "cinéma d'art » ou du «film esthétique ». Pourtant, Léon Gaumont ne va certainement pas s'entendre - en tous points du moins - avec ses contemporains qui prophétisent la naissance de l'art cinématographique, et la rupture radicale avec le répertoire des cafés-concerts et des spectacles populaires. Rétrospectivement, l'échec (relatif) des phono-scènes peut s'expliquer par ce choix malencontreux d'un répertoire peu apprécié dans le cercle des défenseurs des films d'art, lesquels sont exactement contemporains de la publication des Catalogues Gaumont pour Projections Parlantes. L'hypothèse mérite d'être explorée, à plus forte raison le fait que l'idée même de films synchronisés obtient de nombreux suffrages auprès des plus fervents partisans d'un art cinématographique, dont Ricciotto Canudo, «l'inventeur du Septième Art ».

Malgré certaines apparences parfois trompeuses, le cinématographe prend manifestement ses distances à l'égard des pratiques spectaculaires. Du moins, le cinématographe d'art, dont parlent Georges Dureau, Edmond Benoît-Lévy ou encore Ricciotto Canudo, qui doit emprunter une voie radicalement opposée à celle de la "cinématographie-attraction " et des vues trop marquées d'un héritage cabaretier, forain ou, plus généralement, populaire. Peut-être cette rupture est-elle particulièrement manifeste dans le cas précis des Projections Parlantes Gaumont, dans la mesure où aucun des plus ardents défenseurs d'un art du cinéma ne manifeste d'hostilité à l'encontre de la sonorisation de scènes filmées. Or le répertoire des phonoscènes semble quant à lui, a priori, problématique, du moins eu égard aux aspirations de ceux qui soutiennent l'avènement d'un art cinématographique. L'apparente vulgarité de certaines phono-scènes Gaumont ternissent par avance la réputation d'un art en quête d'identité et particulièrement soucieux de protéger son «image de marque ». Symptôme de cette émancipation peut-être en devenir, empreinte d'un discours dont l'objectif est d'effacer, vaille que vaille, les traces d'un passé récent, d'aucuns privilégient une terminologie qui vise à asseoir la légitimité sociale et culturelle d'une pratique aux velléités artistiques. Désormais, nombreux sont ceux qui préfèrent parler de films d'art, plutôt que de scènes filmées.

5 Dès septembre 1908, Dureau va dans ce sens lorsqu'il dresse un premier bilan de la courte histoire du cinématographe. Pour le directeur de Ciné-Journal, les spectateurs, encore sous le charme presque hypnotique de l'invention de la photographie animée, se seraient jusqu'alors satisfaits «des facéties d'un Toto Gâte-Sauce, des poursuites 
effrénées mises à la mode par la maison Pathé, des drames servis en abondance, des féeries diverses et plus ou moins coloriées et des comédies et des pièces de fantaisie et de voyages et des actualités $»^{3}$. Pour Dureau, ce temps est bel et bien révolu car, désormais, « les spectateurs s'érigent en critiques », et sont attentifs au jeu des acteurs et à la qualité de la mise en scène. «Il n'est pas rare », écrit-il, « d'entendre dire "Dieu que c'est mal joué !", ou "mal mis en scène"... » Deux mois à peine avant la sortie de l'Assassinat du Duc de Guise, Dureau ne cache pas son goût pour les "vues réelles »4.

Comique délicat bien joué, actualités, décors et scénarios soignés, voilà le pays du silence cinématographique dont rêve en juin 1909 le directeur de l'Organe hebdomadaire de l'Industrie cinématographique. Sans dénigrer «les mélodrames à grosse sensation, les pièces chères au Grand Guignol, les vaudevilles plaisants ou la pièce policière [qui] entre brillamment au Cinéma avec les aventures de Nick Carter, roi des détectives", Dureau reconnaît ne guère porter dans son cœur le comique facile, les poursuites effrénées ou les féeries, bref « la cinématographie de composition [qui] marche avec la mode, à la façon des revuistes de nos music-halls [et] s'inspire des goûts passagers de la vie parisienne dont elle devient peu à peu comme une manifestation expresse, aussi fugitive $»^{5}$. Dureau accepte bien quelques écarts, mais refuse assez radicalement les films qui conservent des liens de parenté avec le monde du spectacle, du music-hall et du café-concert. À cette " cinématographie de composition", aux attractions et aux films qui conservent des affinités trop grandes avec les représentations des foires et des cafés-concerts parisiens, Dureau préfère désormais le Cinéma. Fustigeant sans relâche la veulerie des "théâtres de bas étage ", il ne déroge jamais aux principes d'une ligne éditoriale fixée par lui dès le premier numéro de Ciné-Journal.

7 À la mi-janvier 1910, le journaliste rédige un éditorial qui reprend quelques-uns de ses chevaux de batailles journalistiques, où s'entremêlent tout à la fois des considérations d'ordre moral ou esthétique (« le coloriage à la machine, les virages et les teintages ajoutent à la valeur esthétique du film ", écrit-il notamment), et des préoccupations qui touchent directement à l'économie du cinéma. Dans ce texte où Dureau emploie cette désormais fameuse expression de «spectateur ordinaire du Cinéma», il est précisément question, en priorité, de «soigner le public, précieux réservoir de la fortune de l'industrie dorée du cinéma». Dans ce dessein, un double préalable s'impose: il importe, semble-t-il, de refuser catégoriquement l'avilissement des programmes et le promouvoir les « effets artistiques »:

Les spectateurs ordinaires du Cinéma qu'on retrouve chaque semaine dans les salles de projection ont été depuis un an mieux servis que jamais. Le prix des places n'a pas augmenté, le confortable s'est accru dans presque toutes les grandes installations et la qualité des programmes s'est fortement améliorée. Il nous faut féliciter les fabricants d'abord qui, selon la loi féconde de la concurrence, ont rivalisé d'efforts artistiques et de soins matériels pour amener leur production à un meilleur titre. Tout le monde doit reconnaître que le film est en progrès. Malgré la pénurie de sujets, hélas, désolante, il reste que les scénarios écrits par des hommes de goût ou des auteurs professionnels sont de mieux en mieux traités par les metteurs en scène; que les décors, les costumes, le cadre historique de l'action rentrent enfin dans la vérité; que les interprètes, après de déplorables essais comprennent l'expression cinématographique et ne se livrent plus - ou rarement à cette épilepsie spéciale dont beaucoup de gens ont pu dire qu'elle était la cinématographie elle-même. ${ }^{6}$

Dans les années dix, l'accompagnement sonore des films préoccupe donc les journalistes, les musiciens et les compagnies inquiètes du sort d'une production 
largement tributaire des exploitants. À cette époque, la presse - dont on ne soulignera jamais assez les liens particuliers qui la lient à l'industrie naissante - commence tout doucement à inscrire la musique au rang des sujets d'actualité d'un cinéma en pleine mutation. L'intérêt porté à la musique au cours de la seconde époque du cinéma est certainement symptomatique des bouleversements qui l'affectent alors en profondeur. Les dissemblances parfois importantes entre les pratiques sonores permettent aux journalistes de convenir qu'un peu d'ordre s'impose. Pour remédier à une telle confusion, qui touche aussi bien la France que les États-Unis, il est alors souvent question de l'harmonisation de la musique de film, et de l'harmonisation du film par la musique. Ciné-Journal, le Moving Picture World ou le New York Dramatic Mirror réservent à cet effet plusieurs de leurs colonnes à la musique de film; en ce sens, Edison, Pathé ou Vitagraph accompagnent leurs films de recommandations plus ou moins précises; certains suggèrent que la musique soit tout à la fois le principal vecteur de l'éloignement du cinéma de pratiques populaires et l'alliée supposée d'un cinéma narratif de qualité; etc. Très clairement, comme le dit Rick Altman, «l'accompagnement sonore devient partie intégrante de la production ».

Aujourd'hui perçue comme la marque d'une vraie richesse, l'extrême diversité des pratiques sonores du «cinéma muet »- variant d'un pays, d'une région, d'une salle à l'autre - est très tôt la cible de critiques acerbes. Des spectateurs vouent aux gémonies les piètres orchestres ou les pianistes médiocres qui sévissent dans les salles où ils interprètent, souvent en dépit du bon sens, des airs à la mode et des chansons populaires sur des drames historiques ou des scènes religieuses 7 . Le 29 juillet 1911, en France, Dureau signe dans Ciné-Journal un éditorial sans complaisance à l'égard des pratiques sonores du cinéma. Dépité par une utilisation grossière de l'accompagnement musical, il remet en question la présence systématique de musique dans les salles de cinéma, où elle est le plus souvent cantonnée au rôle d'appât, d'attrape-spectateur. Lorsqu'ils regrettent que certains exploitants se sentent obligés d'adjoindre des spectacles musicaux aux films, pour en accroître l'attrait, Clyde Martin, Georges Dureau et Vachel Lindsay ne disent pas pour autant que la musique du cinéma constitue un encombrant héritage des bouis-bouis ou des music-halls ${ }^{8}$. Au contraire, pour le rédacteur en chef de Ciné-Journal et les critiques américains, la musique est potentiellement bénéfique à l'art cinématographique. Martin défend la présence de musique dans les salles en ce qu'elle serait incompatible avec la chanson roturière: selon lui, l'accompagnement musical des films doit plutôt chasser des salles de cinéma les chansons issues des cabarets populaires. De son côté, en juillet 1911, Dureau, qui est dépité de leurs usages, suggère assez explicitement qu'on mette fin aux pratiques sonores des premiers temps du cinéma :

La musique est une gouvernante française qui ne suit pas sa jeune maîtresse comme une ombre, mais la laisse se promener selon ses caprices pour n'intervenir que lorsqu'elle est nécessaire :

il ne convient pas qu'on l'entende sans cesse. Elle serait insupportable. [...] Beaucoup d'exploitants cinématographistes, peu confiants dans leurs propres spectacles, se croient obligés pour augmenter l'attrait des films, de les accompagner par quelque musique - piano, phonographe ou orchestre. ${ }^{10}$

Par là, il ne rejette nullement la présence de la musique dans les salles de cinéma. Pour l'éditorialiste de Ciné-Journal, «le principe d'émouvoir la sensibilité des spectateurs par la phrase mélodique est excellent en soi ». Afin d'éviter tout malentendu, qui pourrait lui faire dire qu'il faut bannir des salles le musicien ou le phonographe, il concède 
volontiers : «nous savons tous qu'une scène cinématographique soulignée par le chant d'un violon prend un caractère spécial qui ajoute à sa valeur ", et "qu'un accord bien placé fera plus pour rehausser le film qu'un accompagnement sur tout le métrage ». Sans prétendre mesurer l'impact exact des propos de Dureau - qui tient alors solidement les rênes de Ciné-Journal depuis trois ans -, sa ligne éditoriale paraît en adéquation assez parfaite avec une certaine idée du cinéma en train de se constituer. Lorsqu'il prêche pour un art cinématographique comme d'une possible addition du Cinéma et de la Musique d'Accompagnement, Dureau formule, non sans une certaine prémonition, ce qui peut apparaître aujourd'hui comme la future ligne de conduite des grands studios sur un usage ad hoc de la musique de film :

Il faut - et la condition est nécessaire - qu'il y ait harmonie entre l'expression musicale et le sens du film. Il faut que les tons mineurs appellent nos larmes à la minute même où nous sommes émus par une situation pathétique : sinon l'effet cherché n'est pas obtenu et l'impression générale est faussée. Le spectateurauditeur, balancé entre deux suggestions, qui ne s'accordent plus, regarde mal l'orchestre : il s'agace et, sans se rendre compte parfois pourquoi, s'ennuie. Soyez certains qu'il perdra le goût du cinéma. ${ }^{11}$

Dureau regrette le recours à « d'infâmes pots-pourris plus ou moins mal adaptés et d'un effet artistique intolérable». L'éditorialiste pense sans doute au Film d'Art quand il souhaite des partitions spécifiquement écrites pour les films. Incontestablement, les propos du rédacteur en chef de Ciné-Journal s'accordent avec ceux qu'un Adolphe Brisson tenait fin 1908 à l'occasion de la sortie de l'Assassinat du Duc de Guise. Le chroniqueur $\mathrm{du}$ Temps, jusqu'alors un des plus sévères pourfendeurs $\mathrm{du}$ Cinématographe, se montre en effet particulièrement élogieux pour la musique de Saint-Saëns ${ }^{12}$. Tous deux s'entendent sur l'importance d'une musique de qualité pour la bonne tenue d'un film. Déplorant amèrement les notes discordantes des orchestres, «comme ce trombone et ce cornet à pistons qui, pour achever leurs cartons, continuaient à souffler un air triomphal pendant qu'une petite fille allait mourir sur l'écran », Dureau suggère que l'accompagnement musical soit - faute de mieux - dévolu à un « pianiste improvisateur » :

Le piano est l'instrument par excellence des cinémas-théâtres. Il permet de suivre l'esprit changeant des scènes et de l'exprimer sans retard. [...] Contentons-nous d'un bon pianiste accompagnateur, capable de cacher sa virtuosité professionnelle et assez artiste pour n'intervenir qu'aux minutes « musicales $» .^{13}$

Le journaliste conclut qu'en l'absence d'un tel collaborateur, le cinéma a tout intérêt à laisser au film "son caractère essentiel qui est d'être une comédie ou un drame ${ }^{14}$. Quelque deux ans plus tard, Dureau réserve en avril 1913 les colonnes de sa revue au chef d'orchestre de l'Eden Cinéma Pathé de Marseille. Si une telle faveur est accordée à ce dernier, c'est bien parce qu'il « s'élève contre le mauvais goût de quelques-uns de ses collègues en matière d'accompagnement cinématographique $»^{15}$. En substance, les regrets du chef d'orchestre marseillais s'accordent avec les propos tenus par Dureau en juillet 1911. Les deux hommes clament en chœur que « les orchestres se chargent bien souvent de ridiculiser une vue en jouant des morceaux qui ne s'adaptent pas avec l'action $»^{16}$. Enfin, comme Georges Dureau quelques mois plus tôt, le musicien admet que si l'écriture de partitions spéciales n'est apparemment pas d'actualité, "on pourrait tout au moins prendre la peine de chercher à adapter quelques morceaux ou fragments de morceaux s'accordant un peu mieux avec la vue et en soulignant les passages caractéristiques $»^{17}$. 
13 Néanmoins, Dureau et ses collègues sont rarement prolixes sur ce qu'ils entendent par «musique appropriée ». En dehors de la compétence de l'orchestre, il n'est pas aisé de cerner ce qu'ils attendent d'un musicien et d'un compositeur. Ils ne s'expriment guère sur le type de musique, ou sur le nom éventuel d'un auteur. Sans doute songent-ils à une musique grandiose, digne du septuagénaire Camille Saint-Saëns et de sa partition de l'Assassinat du Duc de Guise? Dureau demande juste une musique de qualité jouée par des professionnels, une musique qui ne laisse plus les films sous la férule d'interprètes occasionnels et d'exploitants peu scrupuleux. Pour le rédacteur en chef de Ciné-Journal, une condition est nécessaire et suffisante à la bonne tenue artistique du film et de sa musique : « Il faut qu'il y ait harmonie entre l'expression musicale et le sens du film ». Il proscrit par avance le moindre écart, de quelque sorte, entre la musique et le film. D'une grande pondération, Dureau refuse les mauvais accords, et ne privilégie rien d'autre qu'une musique docilement asservie aux images animées.

Aux alentours de 1912, en France comme aux États-Unis, les sociétés éditrices semblent avoir comme préoccupation une certaine mainmise sur un produit fini. Les musiciens sont à cet effet de plus en plus souvent délaissés au profit de pianos ou d'instruments mécaniques, au demeurant fort appréciés du public ${ }^{18}$. À l'époque, un certain John B. Rathbun soutient qu'avec un piano mécanique l'exploitant peut faire de substantielles économies, et éviter tout conflit avec les puissants syndicats des musiciens ${ }^{19}$. Sous le couvert de bonnes intentions, où il est surtout question de défendre les qualités artistiques des films, des raisons économiques et politiques avivent manifestement les débats. La perspective de graver l'accompagnement sonore d'un film est alors une alternative à l'incompétence notoire de prétendus musiciens, à d'éventuelles revendications syndicales, à l'exploitation peu scrupuleuse des films et au manque de docilité des propriétaires de salles à l'égard des injonctions des compagnies éditrices.

En janvier 1912, alors que la "phono-cinématographie » semble avoir ses beaux jours derrière elle, un journaliste consacre un long article au procédé de synchronisation d'un certain Louis Janssens, obtenu au moyen d'un pianola et du cinématographe ${ }^{20}$. Ce texte constitue un argumentaire implacable qui énumère toutes les bonnes raisons artistiques et commerciales en faveur de la «synchronisation MusicoCinématographique ». L'article n'est pas un billet d'humeur, et il est très loin de fuir l'actualité économique, juridique et esthétique du cinéma. Le texte insiste beaucoup sur les bénéfices financiers à retirer de la synchronisation. Pour l'auteur du dossier, celle-ci affermirait sans aucun doute la mainmise de l'éditeur du film sur les rouages de la distribution, sans pour autant gruger le signataire du brevet du procédé sonore. L'idée directrice du dossier est de proposer aux grandes compagnies qu'elles s'adjoignent les services d'un inventeur compétent qui leur fournirait le système d'enregistrement et les rouleaux ou les disques gravés par ses soins :

Cette collaboration consiste simplement en l'autorisation à donner à l'inventeur par l'éditeur de films, d'improviser et laisser improviser chez lui, par ce premier ou un ou plusieurs pianistes compositeurs désignés par ledit inventeur à chaque passage d'un film nouveau chaque semaine, sa ou leurs improvisations devant être enregistrées sur un rouleau à l'aide d'un appareil ad hoc, lesquelles improvisations seraient reproduites en un nombre égal d'exemplaires à celui du nombre de films édités chaque fois, et qui pourraient au besoin être vendus ensuite par l'éditeur même, en même temps aux acheteurs, loueurs, etc., que ses films. L'inventeur, d'accord avec une ou plusieurs maisons fabriquant ces instruments reproducteurs s'occupera de la vente des pianos reproducteurs dans les cinémas et de leur 
accouplement synchronique aux appareils cinématographiques, objet de ses brevets. $^{21}$ que court le théâtre cinématographique dans l'avenir à laisser abîmer le spectacle des yeux par une audition non appropriée de la musique ». Cette dernière partie du dossier dit bien que «tous les cinémas seraient obligés d'adopter le système ». Face aux périls que le cinéma encourt, il n'est plus question de tergiverser, ou de discuter au coup par coup avec les exploitants, mais bien d'adopter une procédure assez autoritaire. Pour justifier de telles injonctions, le journaliste pointe du doigt ce qui, depuis l'Assassinat du Duc de Guise, motive quelques-uns des promoteurs de l'art cinématographique à souhaiter avec une telle conviction l'accompagnement musical des films. Manifestement, la synchronisation musico-cinématographique peut conduire le cinéma sur le chemin de la standardisation des modes d'exploitation et de consommation des films :

Le théâtre cinématographique, rendu ainsi artistique à tous points de vue deviendra un vrai théâtre de l'art. Il ne se fera plus de commentaires désobligeants dans les milieux qui se piquent de mondanité et de snobisme, contre la musique telle que, et pour cause, elle est donnée dans le cinéma, ni par le fait, contre le cinéma luimême. Or cette attitude, cette propagande est néfaste au cinéma de l'avenir. Toute une classe nombreuse, celle qui marque le pas aux autres, la classe mondaine, ne va que timidement, en cachette, presque honteusement, au cinéma. Pourquoi ? Parce qu'il manque à celui-ci précisément cette chose qui imprime à ce spectacle le vrai cachet artistique ; cette chose, l'art musical y approprié. ${ }^{22}$

Dans cette optique, la musique synchronisée constitue la planche de salut de l'art et de l'industrie cinématographiques. Préalablement bien choisie, fournie avec le film, la musique réglée synchroniquement maintiendrait le cinéma sur les droits chemins culturel et industriel, entre les ornières tracées par le Film d'Art en France. L'auteur du dossier de Ciné-Journal est particulièrement clair sur ce point :

Ni la beauté du film, ni la beauté des salles, ni la profusion des lumières pendant les interruptions n'auront raison de cette bouderie, quasi obligée de la classe aisée, de son dédain obstiné. Que la musique soit réglée synchroniquement au cinéma, le régal d'art n'y sera plus discutable ; les mondains et les férus d'art fréquenteront le cinéma, c'est ce qui y amènera le vrai succès constant et en fera le grand théatre de l'avenir. La réciproque n'est pas moins vraie, et s'il n'est pas réagi de telle sorte contre la mauvaise qualité donnée aux spectacles cinématographiques, par la musique, les meilleurs théâtres cinématographiques au lieu de continuer à progresser et à être le grand théâtre de l'avenir, deviendront de plus en plus le théâtre ravalé du populaire, à part quelques exceptions de circonstances peut-être. 23

18 À première vue, lorsque Dureau, tel autre journaliste de Ciné-Journal et tel chroniqueur américain exigent un pianiste accompagnateur, une musique appropriée ou les notes d'un violon, c'est d'abord pour compléter l'émotion des images. L'accompagnement musical suppléerait à un certain vide laissé par les images animées, et, par la même occasion, estampillerait le cinéma d'un « cachet artistique ». La musique accorderait en effet au cinéma ses lettres de noblesse culturelle, pour mieux satisfaire un public cultivé qui boude, au grand dam de Dureau, les salles de cinéma. En tout état de cause, la musique prend là les atours d'un faire-valoir esthétique qui accorde implicitement à l'industrie la mainmise sur ses produits. Les prises de position de Dureau sur la musique de film (ré)concilient, non par hasard, les deux missions qu'il s'est fixées depuis 1908 au lancement de Ciné-Journal. Lorsqu'il endosse son habit de redresseur de torts des 
accompagnements mal venus, il joue en effet à la fois le rôle de «commissionnaire de l'acheteur " et de critique cinématographique, c'est-à-dire à la fois celui de porteparole des compagnies et celui de guide du public. Menant une campagne pour standardiser le son, le commissionnaire de l'acheteur et le critique défendent d'une seule voix les intérêts de l'Art et de l'Industrie.

Des années dix aux années vingt, le cinéma va plutôt tout mettre en œuvre pour pouvoir se passer de la parole, et sacrifier les «scènes chantées » et les Projections Parlantes, désormais prises entre les feux contradictoires du spectacle et de l'art cinématographiques, du cinéma institutionnel et du Septième Art. À n'en pas douter, les écrits de Canudo sont pris dans ce courant où refluent des textes animés d'un même souci: légitimer l'art cinématographique. Alors que Maugras et Guégan, malgré quelques détours sur les à-côtés de l'esthétique et de la morale, investissent le champ juridique, tandis que Dureau et Edmond Benoît-Lévy, un premier temps les intermédiaires entre l'acheteur et les compagnies éditrices, jettent les bases d'une tradition critique française de haut vol, Canudo élabore entre 1908 et 1923 un discours qui a, lui, les allures d'une pensée théorique ${ }^{24}$. Au fil du temps et de ses articles, Canudo sera rejoint par Delluc, Dulac, Epstein et d'autres grands noms - aux fortes consonances européennes - de la théorie du cinéma des années dix et vingt. Condensée en une quinzaine d'années, l'œuvre de Canudo, qui décède le 10 novembre 1923, est d'une densité relativement mésestimée. Dès ses premiers textes, où il exhorte au triomphe du cinématographe et clame la naissance prochaine d'un art nouveau, sa réflexion marque immédiatement les esprits d'intellectuels, comme ses amis Apollinaire et Fernand Divoire, alors une des plumes les mieux acérées de Phono-Ciné-Gazette $e^{25}$.

Pourtant, l'œuvre théorique de Canudo ne se résume pas à quelques textes écrits vers 1910, dont on dit partout qu'ils sont, dans un même élan, fondateurs du septième art et de la théorie du cinéma. En quinze ans, Canudo écrit une centaine d'articles dont l'objet commun est la définition d'un Art plastique en mouvement. Une extrême circonspection s'impose quand il s'agit d'analyser ses écrits, très rigoureux, car le cinématographe dont il parle en 1908 n'a pour ainsi dire que peu à voir avec le Cinéma qu'il défend dans les années vingt. Du moins, l'état du cinéma (tographe) n'est-il pas exactement comparable. Le fameux "Triomphe du cinématographe", publié à Florence le 25 novembre 1908, laisse à penser qu'il est assez conciliant à l'égard de ce qu'il désigne encore par spectacle cinématographique. En 1908, il accorde un sursis aux divertissements en tous genres, des scènes comiques aux spectacles phono-cinématographiques ${ }^{26}$. Reconnaissant au Cinématographe le droit de divertir et de conserver sa dimension spectaculaire encore quelques temps, le répit sera de courte durée. Décidément, les phono-scènes du Catalogue pour Projections Parlantes n'avaient rien, ou très peu, pour plaire aux inventeurs de l'art cinématographique et du cinéma muet. "Le triomphe du cinématographe » n'en annonce pas moins le Drame musical ou le film musical, loin des spectacles des foires et des cabarets, autant dire une production, non pas chantante ou parlante, mais plutôt complétée d'une musique de film.

21 Dans les années dix et vingt, Canudo use de sa verve pour convaincre son lecteur de la faible intelligence et du manque de raffinement des classes populaires, incapables, selon lui, d'apprécier les nuances des compositions musicales contemporaines qui doivent accompagner le mouvement des images. En quelques années, Canudo va exclure radicalement tout ce qui, de près ou de loin, peut entacher l'art cinématographique de séquelles populaires et de pratiques spectaculaires appréciées de 
toute classe. Dans un exercice périlleux, il énumère ce dont le Cinématographe doit, selon lui, se défaire s'il veut être à la hauteur de ses ambitions artistiques. Si le «théâtre de Pantomime nouvelle » ou le " Théâtre Cinématographique » disparaissent de son vocabulaire, les «scènes les plus tumultueuses, les plus invraisemblablement mouvementées» et le «spectacle paroxystique de la vie extérieure» du Cinématographe ne bénéficient plus de ses faveurs. Les « spectacles très comiques » et les "apparitions extravagantes» ou les "transformations de mouvements et de figurations » vont peu à peu, au fil de ses écrits, décroître dans son estime au profit du Drame musical et du Septième Art.

D'autre part, Canudo n'a certainement pas comme premier souci de « contrôler l'espace sonore " pour d'obscures motivations économiques. Si d'aventure son objectif est de tendre in fine vers une certaine standardisation des pratiques cinématographiques, c'est plutôt pour atteindre un idéal artistique. Au contraire d'un journaliste comme Dureau, il n'a cure des préoccupations de l'industrie et des « commerçants du cinéma ». Chez lui, le cinéma est cosa mentale, non cosa industriale. Pour le poète, le Septième Art doit opérer une double rupture. Dans un premier élan de sécession, il doit s'arracher des pratiques populaires auxquelles l'histoire a lié son destin, à peine sorti des usines lyonnaises. D'un autre côté, le cinéma ne doit pas nouer des liens de dépendance, sous quelque forme, avec l'industrie du cinéma. Pour Canudo, le cinéma est d'abord un art majeur ${ }^{27}$.

Les débats contradictoires sur l'union de la musique et du cinéma vont longtemps engager des musiciens, des réalisateurs, des mélomanes, des journalistes et des artistes. En France, l'entente paraît souvent belle entre ce que le cinéma possède de plumes plus ou moins affûtées. Les critiques, les artistes et les théoriciens du cinéma s'entendent apparemment au moins - sur ce point : la musique doit conduire le cinéma sur la voie royale du septième art, et définitivement extraire le cinématographe du bourbier cabaretier et forain où il s'est, à leurs yeux, malencontreusement empêtré dès son plus jeune âge. Chez Dureau ou Canudo, la musique doit donc émanciper le cinéma d'art de ses racines cabaretières, où effectivement un ou plusieurs instrumentistes agrémentaient souvent les spectacles et les intermèdes ou les entractes. Pour Dureau et Canudo, la musique doit visiblement marquer une rupture avec le " désordre sonore " des salles de projection des premiers temps du cinéma. Quand l'un et l'autre exigent une musique, présente à des moments opportuns, pensée et écrite pour le film, ils tentent bien de casser l'hétérogénéité du dispositif de présentation et de consommation des films de la première époque, pour davantage tendre vers l'homogénéité plus marquée du mode de (re)présentation de la seconde époque du cinéma.

Dans le milieu des années dix, un nouveau modus vivendi tente de s'imposer. Par égard pour ce nouveau mode de consommation, la musique va désormais suivre au plus près le film, pour se détacher progressivement des réactions aléatoires des spectateurs des cinémas. Après avoir assuré une part de l'ambiance des salles avant, pendant et (ou) après les projections, la musique tend désormais à se mettre entièrement au service du film, pour coloniser son espace diégétique. Dès le milieu des années dix, tel « un ombilic faisant fonction ", elle nourrit le spectateur ordinaire du cinéma de l'émotion des images animées. L'accompagnement musical des films, souhaité par plusieurs compagnies éditrices et recommandé par quelques-uns de leurs relais dans la jeune presse cinématographique (Ciné-Journal, Phono-Ciné-Gazette), est promis à de beaux 
jours. La partition de l'Assassinat du Duc de Guise les convainc de persévérer sur cette voie. Alors que le Film d'Art paraît fédérer les aspirations artistiques du cinéma(tographe), tandis que la musique semble constituer le point de ralliement de tous ceux qui souhaitent définitivement accorder aux vues animées leurs lettres de noblesse culturelle, l'accompagnement musical est vite le point d'achoppement de la belle entente qui paraît alors régner entre tous les amateurs déclarés de l'art cinématographique, de Dureau à Canudo. Le mariage de raison convié par l'un, où la musique est littéralement au service de la standardisation du film (à la fois l'objet d'une «production de l'esprit » et d'une consommation spectatorielle), ne s'accommode pas vraiment avec la fusion complète de la musique et de l'art cinématographique en un Septième Art prêchée par l'autre. Cette dissension va, au fond, mettre à nu les motivations profondes qui, dès les années dix, poussent les uns et les autres à exhorter de concert à l'avènement d'un hypothétique art du cinéma. Les désaccords de prime abord imperceptibles entre Dureau et Canudo sur la présence musicale au cinéma présagent certainement de quelques-uns des plus sérieux débats, des années dix aux années vingt. La distinction entre " accompagnement musical » et «film musical » se révèle au fil du temps un point de rupture radicale entre les chantres de la standardisation des pratiques cinématographiques et les partisans du Septième Art, lesquels, pour la plupart, n'auront de cesse de vouer aux gémonies le rôle à leurs yeux néfaste du Film d'Art en général et de l'Assassinat du Duc de Guise en particulier.

Une vingtaine d'années plus tard, l'histoire semble se répéter, avec une issue radicalement divergente, quand la Warner lance sur le marché américain des centaines de courts métrages dont le répertoire n'est pas sans rappeler celui des phono-scènes Gaumont. La production des Vitaphone Shorts, qui se résume pour l'essentiel à deux grands registres, est en de nombreux points similaire à celle des «films chantants et parlants » Gaumont : les scènes filmées des spectacles des cafés-concerts et les extraits d'Opéras pour l'une, et les spectacles de Broadway et les livrets opératiques ou les grands classiques musicaux de la musique occidentale pour l'autre. Si les extraits d'opéras, de sonates ou de symphonies doivent constituer, dans un premier temps, le répertoire privilégié par la Warner, très vite, les vaudfilms n'en font pas moins une entrée fracassante dans les catalogues. Immanquablement, quand il s'agit de prendre le pouls des films parlants à la fin des années vingt, cette ressemblance déplacée - selon l'expression de Georges Didi-Hubermann à propos de tout autre chose - permet certainement de mieux percevoir l'état du cinéma peu de temps avant la consécration définitive du cinéma parlant.

Le 6 août 1926, le premier programme complet de Vitaphone Shorts est présenté au Warner's Theatre de New York, en prélude à la projection de Don Juan. Le programme comporte huit films (de trois à onze minutes), enregistrés entre le 17 juin et le 22 juillet $1926^{28}$. Pour beaucoup d'historiens, la soirée du 6 août 1926 est à marquer d'une pierre blanche, car la première de Don Juan serait le prélude à la "révolution du parlant ». Avant d'y revenir, peut-être faut-il rappeler, d'une part, que la Warner ne songe pas encore à évincer le "cinéma muet » au profit du « cinéma parlant », et, d'autre part, que la compagnie n'agit pas en franc-tireur. Certaines de ses rivales, comme la Fox, s'engagent elles aussi sur cette piste d'un "cinéma sonore ». De plus, les industries de la radio et du téléphone sont aux abois, conscientes des intérêts en jeu, et prêtes à lier leur destin à plusieurs compagnies cinématographiques. Entre autres pour ces raisons, rien ne permet d'affirmer qu'en 1926 ou 1927 le muet va chasser le parlant, a fortiori d'un revers de main. Au contraire, les déclarations des frères Warner vont plutôt dans 
le sens d'un "cinéma sonore ", où une musique et des bruitages accompagneraient un long métrage. Durant ces années, la parole est clairement réservée aux scènes chantées et parlées des sketches qui reproduisent «à l'identique» les spectacles, souvent bavards, presque toujours chantés, de Broadway. À l'instar de Gaumont qui louait vingt ans plus tôt la nouveauté de son Chronophone, Harry Warner adresse une lettre aux exploitants pour leur faire part de la nouveauté et de la qualité du procédé Vitaphone. Publié le 24 juillet 1926 dans Brass Tacks, un feuillet édité par la Warner Bros., le courrier privilégie l'accompagnement musical des films, en aucun cas l'éviction du « muet » ou l'avènement du « parlant " ${ }^{29}$.

En aucune manière, The Jazz Singer (le Chanteur de jazz) n'est lancé en éclaireur. Le Vitaphone Warner fait ses preuves dès avant la sortie du film d'Alan Crosland. Le catalogue Vitaphone est riche de centaines de titres - courts ou longs métrages - avant « la naissance du cinéma parlant ». Par ailleurs, en cette fin des années vingt, The Jazz Singer ne peut a priori faire de l'ombre à Don Juan, où John Barrymore, star incontestée $\mathrm{du}$ box-office hollywoodien, excelle dans son rôle de chevalier séducteur ${ }^{30}$. Don Juan constitue de la sorte la première tête d'affiche des longs métrages Vitaphone, et non un banc d'essai qui mènerait à leur hypothétique point culminant, The Jazz Singer. La face cachée de cet iceberg est en effet constituée de centaines de Vitaphone Shorts sortis des studios de la Warner dès 1926. Aujourd'hui, on le sait, débute alors une période qui va mener à la généralisation du cinéma parlant. À l'époque, rien n'est moins sûr. C'est donc certainement dans cet entrebâillement, entre lesdits " premier film sonore » et "premier film parlant» de l'histoire du cinéma, qu'il y a lieu de progressivement s'engouffrer. Au propre comme au figuré, cet interstice de l'histoire du cinéma est problématique.

Le 6 août 1926, un message de Will H. Hays, Président de la Motion Picture Producers and Distributors of America inaugure cette soirée exceptionnelle, au cours de laquelle seront présentés huit courts métrages Vitaphone et le très attendu Don Juan. Dans Hon. Will. H. Hays welcomes Vitaphone in an Adress, Hays se présente face à la caméra. Il est debout devant un bureau, avec à l'arrière-plan un rideau noir sur lequel se distinguent entrecroisées deux lettres (V/C) à l'effigie de la Vitaphone Corporation. Par ce discours, Hays salue les efforts des frères Warner, et, sur un ton solennel, consacre l'avènement du film sonorisé. Le discours a certainement pour double objectif de faire la preuve de la fiabilité d'un système d'enregistrement et de dessiner les contours du cinéma sonore des années trente. Dans son discours d'introduction, Hays imagine un cinéma où la musique synchronisée va jouer un rôle inestimable, et des films où la «bonne musique » et l'art lyrique iront à la rencontre des hameaux les plus reculés. Dans son speech, il n'est jamais question de cinéma parlant. A posteriori, les discours de Hays et de Sam Warner témoignent, bien malgré eux, des incertitudes de l'industrie américaine du cinéma quand, dans un premier temps, celle-ci semble plutôt croire au "film sonore » (pour diversifier la production « muette »). Le choix, peu à peu unanimement reconnu, d'un procédé de reproduction de "son-sur-film » n'est pas vécu comme une révolution. Il n'en est pas moins déterminant, dans la mesure où il participe d'un lent processus mis en œuvre de longue date, c'est-à-dire une certaine standardisation du cinéma. La musique d'accompagnement - synchronisée, collée, assujettie aux images - participe largement à détourer le profil d'un cinéma classique parlant en plein essor.

Rétrospectivement, on comprend dès lors pourquoi les attentes des uns et des autres ne peuvent s'accorder lorsqu'il est de plus en plus question de synchroniser le son et 
l'image à la fin des années vingt. Sans doute Don Juan, qu'une musique suit comme son ombre, constitue-t-il l'aboutissement d'un accompagnement musical «fidèle aux émotions des images » et au déroulement narratif du film. Dans le même temps, les héritiers de Canudo - du moins certains d'entre eux - ne se réjouissent pas de la tournure des événements, et s'accordent pour les uns défendre le "film sonore» et les autres un contrepoint orchestral. L'avènement du "film sonore » consacre le synchronisme idéal du Drame musical de l'Écran tant espéré par Canudo au tout début des années vingt. Plutôt désappointés quant à l'état du cinéma muet, plusieurs des chefs de file des avantgardes françaises ne disent alors rien d'autre qu'Alexandrov, Eisenstein et Poudovkine dans les premières lignes de leur « déclaration » sur le contrepoint orchestral : «Le rêve depuis longtemps caressé du film sonore est maintenant une réalité.» Le «film sonore » serait peut-être ce film idéal. Parmi ceux qui eux attisent la flamme d'un art cinématographique sonore, Germaine Dulac est indubitablement très explicite sur les espoirs que cette génération entretient à l'égard d'un « film parlant idéal » (ce sont ses mots). C'est elle qui récuse le " cinéma parlant et sonorisé », et défend avec la même conviction le "film sonore"; c'est elle qui appelle à un silence ponctué de mots, de cris, de bruits et d'exclamations parcimonieuses; c'est encore elle qui médite " une orchestration synchrone de bruits et d'images » et qui pense le cinéma comme de «la musique par essence $»^{31}$. Voix vs Parole, accompagnement musical approprié vs musique de film, ou encore film sonore vs cinéma parlant et sonorisé, voilà certainement, en deux ou trois équations, ce qui, en cette fin des années vingt, peut opposer radicalement l'industrie du cinéma dans ses contours les plus larges et les réalisateurs en marge de cette même industrie.

The Jazz Singer est un film à tout le moins emblématique de la relation paradoxale entre la musique et le cinéma. En effet, la musique du « premier film parlant de l'histoire du cinéma " passe à ce point inaperçue qu'aucun commentaire n'en dit le moindre mot depuis la sortie du film. Au moins autant que le Parlant, The Jazz Singer consacre un accompagnement musical approprié, sans trop de relief, soumis et fidèle aux images et à la narration classique. En ce sens, Theodor Adorno et Hanns Eisler ont-ils raison de dire que « l'un des préjugés les plus répandus dans l'industrie cinématographique est que la musique n'est pas faite pour être entendue $»^{32}$. Vers 1935, le Parlant entérine la suprématie de l'accompagnement musical approprié du cinéma institutionnel, et marginalise définitivement le Drame musical de l'Écran et le «film sonore ».

\section{NOTES}

1.Je me permets de renvoyer le lecteur à trois de mes articles où il est déjà question de la musique des films muets et des spectacles phono-cinématographiques : « Del mudo al parlante, del café-concierto a la época del jazz », Archivos, n 30, octobre 1998, p. 24-37, "Les doigts dans les oreilles et la majesté du silence. Des "films chantants et parlants", du cinéma muet et des autres arts ", dans Leonardo Quaresima (dir.), La decima musa. Il cinema e le altre arti, Udine, Forum, 2001, p. 459-471 et « L'événement et la série. Le déclin du café-concert, l'échec relatif du Chronophone et la naissance de l'Art 
Cinématographique ", dans Richard Abel et Rick Altman (dir.), The Sound of Early Cinema, Indiana University Press, 2001, p. 279-287.

2.Catalogue Gaumont pour Projections Parlantes, 1908, p. 37.

3.Georges Dureau, « Le Cinéma et le Théâtre », Ciné-Journal, n 5, 15 septembre 1908, p.

$1-2$.

4.Ibid., p. 2.

5.Ibid., p. 1-2.

6.Georges Dureau, « Soignons le public », Ciné-Journal, n 74, 16-22 janvier 1910, p. 1-2.

7.Vachel Lindsay, New York Dramatic Mirror, 5 juillet 1911, cité d'après Rick Altman,

"Naissance de la réception classique. La campagne pour standardiser le son»,

Cinémathèque, $\mathrm{n}^{\circ}$ 6, automne 1994, p. 101.

8.Ibid., p. 110. Ceci devrait nuancer la relation causale suggérée par Noël Burch, qui semble dire que la musique devait immanquablement accompagner les films après le passage du cinéma des origines dans les cafés-concerts. Burch écrit : « La musique n'était pas ce qui avait manqué à Gorki, à qui on avait dû d'ailleurs en servir à satiété. En effet, on était au caf'conc' ! Et pourtant de cette musique, il ne souffle mot : elle est un élément constituant de l'atmosphère factice de ce lieu de perdition, "chez Aumont". Pourtant, ce côté spectacle musical de bas étage, que le film partage avec le numéro de main à main accompagné par l'orchestre, par exemple, restera partie intégrante du cinéma pendant toute l'époque muette, survivance têtue de ses origines dans le spectacle populaire et présentationnel. » (Noël Burch, la Lucarne de l'infini. Naissance du langage cinématographique, Paris, Nathan Université, coll. « Fac. Cinéma», 1991, p. 224.) 9.Clyde Martin, «Playing the Pictures », The Film Index, 24 décembre 1910, p. 28, cité d'après Rick Altman, op. cit., p. 109.

10.Georges Dureau, « Le Cinéma et la Musique d'Accompagnement », Ciné-Journal, $\mathrm{n}^{\circ}$ 153, 29 juillet 1911, p. 1-2.

11.Ibid., p. 1.

12.Dans cet article, Adolphe Brisson note : « M. Camille Saint-Saëns a écrit pour l'Assassinat du Duc de Guise un chef d'œuvre de musique symphonique. Il eût été impardonnable de n'en pas proclamer les beautés... Ce fut une des parties les plus goûtées de cette représentation un peu tâtonnante, imparfaite, mais intéressante ainsi que tout ce qui commence et promet » (Adolphe Brisson, « Ce que M. Brisson pense du film d'art ", Ciné-Journal, n 17, 10 décembre 1908, p. 9).

13.Georges Dureau, « Le Cinéma et la Musique d'Accompagnement », art. cit., p. 2.

14.Ibid., p. 8.

15.Georges Dureau, « L'accompagnement musical des films », Ciné-Journal, n² 244, 26 avril 1913, p. 2.

16.Ibid., p. 8. Le chef d'orchestre ajoute : « Un directeur de cinéma faisait, il y a quelques semaines, chanter une romance sentimentale pendant la projection d'une vue documentaire. Le contraste avec le sujet représenté était d'une ironie toute spéciale ». 17.Ibid., p. 2.

18.Eileen Bowser relève qu'aux États-Unis les exploitants ont largement profité de cette faveur des spectateurs pour écarter des salles des artistes de mieux en mieux organisés, et aux exigences sans cesse accrues. Elle écrit : « Les instruments mécaniques avaient beaucoup de succès. En 1912, quand les musiciens ont constitué une corporation et ont organisé des mouvements de grève pour défendre leurs droits, les exploitants se sont montrés particulièrement prévenants à l'égard des fabricants de musique mécanique 
[mechanical music-makers] ». (Eileen Bowser, The Transformation of Cinema. 1907-1915, New York, Charles Scribner's Sons, 1990.p. 13).

19.Il déclare : « On peut toujours trouver en presque n'importe quel endroit un chanteur ou un pianiste pour accompagner la projection du film, et ce, à un coût modéré. Le salaire de ces gens varie certes considérablement suivant la taille de la ville, les conventions syndicales en vigueur, mais demeure compris entre un et trois dollars par soirée. Si l'on adjoint au pianiste un batteur, il est conseillé de lui verser une somme équivalente, sauf convention syndicale contraire. On peut aussi acheter ou louer dès le départ un piano mécanique ou un orchestrion : c'est un moyen sûr de réduire les coûts dans le cadre d'une petite exploitation. On peut utiliser ces instruments par ailleurs pour produire de la musique dans l'intervalle entre les chansons durant la projection, le pianiste pouvant alors être affecté à d'autres tâches, tenir la caisse ou servir d'ouvreuse. Cette dernière option convient tout particulièrement lorsque l'exploitant fait aussi fonction de pianiste. Le piano mécanique est aussi d'un grand secours lorsque les musiciens viennent à faire fauxbond ». (John B. Rathbun, Comment faire et montrer des films, cité d'après David Robinson, Musique et cinéma muet, op. cit., p. 33.)

20.Louis Janssens, « Synchronisation Musico-Cinématographique », Ciné-Journal, $\mathrm{n}^{\circ}$ 178-179, 20-27 janvier 1912, p. 17-19 et p. 41.

21.Ibid., p. 19.

22.Ibid., p. 19.

23.Ibid., p. 19.

24. Voir : Émile Maugras, M. Guégan, le Cinématographe et le droit, Paris, V. Giard / E. Brière, 1908. Le manuscrit de l'ouvrage est déposé à la Library of Congress de Washington. Une copie de ce document m'a été transmise par André Gaudreault (qui lui a consacré, avec Roger Odin, une communication à Udine en mars 2000).

25.Ricciotto Canudo, l'Usine aux images, Paris, Séguier / Arte Éditions, 1995 [1927].

26.Il n'est pas directement question des phono-scènes dans « Triomphe du Cinématographe ». Par contre, il est clairement fait mention des spectacles phonocinématographiques. Encore une fois, la rhétorique y est subtile, pour dire au fond qu'il est prêt à supporter certaines choses. Plus très longtemps : « Le Théâtre Cinématographique est le premier théâtre nouveau, et, lorsque, comme on le fait déjà d'une certaine manière, il sera enrichi par l'Esthétique et complété par une musique hautement conçue et supérieurement exécutée, même si c'est en représentant complètement la vie réelle avec l'aide du phonographe, on pourra quand même éprouver l'émotion "templaire », le frisson religieux, de la religion à venir [...] Le public moderne est un abstracteur admirable, puisqu'il peut jouir des abstractions les plus absolues de la vie. J'ai pu voir à l'Olympia, à Paris, les spectateurs applaudir frénétiquement le Phonographe qui était sur la scène, habillé de fleurs, et dont la trompe de cuivre venait de sortir un duo de la Favorite... La machine triomphait, le public applaudissait le fantôme sonore des acteurs lointains ou morts. C'est avec un semblable esprit que les foules accourent aux Théâtres cinématographiques qui font partout fureur, et y apportent leur désir de fêtes nouvelles » (Ibid., p. 30).

27.L'attitude du poète italien à l'égard de l'industrie du cinéma contraste avec celle de la plupart des journalistes de l'époque ou de juristes comme Maugras et Guégan qui, à des degrés divers, en défendent les intérêts. En effet, lorsqu'ils écrivent leur ouvrage le Cinématographe devant le droit, rien n'indique que le travail de Maugras et Guégan est commandité par Pathé, même s'il ne va en aucun cas à l'encontre de ses intérêts. Dans 
la mesure où les auteurs du livre ont avec la firme au coq des relations toutes particulières - le premier en sa qualité d'administrateur délégué de l'Omnia-Pathé et de Président du Conseil du Cinéma-Théâtre Pathé, et le second en tant qu'administrateur du Cinéma National Pathé -, un léger doute subsiste quant à leurs aspirations profondes, et aux raisons qui les poussent à défendre avec fermeté un art nouveau. 28.Don Juan est à l'origine un film muet pour lequel un accompagnement a été expressément composé. La musique et les bruitages, comme le tintement des cloches au cours de la séquence du mariage et l'entrechoquement des épées lors de la scène du duel, sont gravés en juin 1926. Les quatre premiers disques sur les treize du film seront réenregistrés le 12 août 1926. Par contre, les disques des Vitaphone Shorts du programme d'ouverture sont enregistrés au cours du tournage. On sait que cette pratique (jouer de la musique pendant le tournage des films) est courante et même recommandée par certains critiques à l'époque du cinéma muet.

29.Harry Warner, «A new Era in Motion Picture... », Brass Tacks, 24 juillet 1926, cité d'après Scott Eyman, The Speed of Sound. Hollywood and the Talkie Revolution 1926-1930, New York, Simon \& Schuster, 1997, p. 89-90.

30.L'histoire du cinéma a conservé un vague souvenir des Vitaphone Shorts, préférant instaurer Don Juan au rang de premier film sonore, en attendant la « tornade » de The Jazz Singer, le premier film parlant. En 1986, Douglas Gomery mettait pourtant en exergue leur rôle majeur dans l'histoire économique de la Warner à la fin des années vingt. Trois ans plus tard, Charles Wolfe rédigeait un des rares essais sur les premiers pas des Vitaphone Shorts et des talking pictures. Les pages ici consacrées aux Vitaphone Shorts doivent beaucoup à ces articles. Pour la plupart disparus ou incomplets, les Vitaphone Shorts analysés ici font tous partie du programme de projection accompagnant l'exposition organisée au MoMA en octobre-décembre 1989. Depuis, des projets de restauration sont en cours, soutenus par des institutions comme le MoMA, l'UCLA ou la Library of Congress ou... Ted Turner. Pour plus de détails, voir : Rick Altman (dir.), Yale French Studies, n 60, 1980 ; Donald Crafton, The Talkies. American Cinema's Transition to Sound (1926-1931), New York, Charles Scribner's Sons, 1997 ; Douglas Gomery, The Hollywood Studio System, New York, St Martin's Press, 1986, p. 104-121; Charles Wolfe, "On the Track of the Vitaphone Short ", dans Mary Lea Bandy (dir.), The Dawn of Sound, MoMA, New York, 1989, p. 35-41.

31.La distinction entre « film sonore » et « film sonorisé » est comparable à celle effectuée ici entre « accompagnement musical » et « musique de film » (ou «film musical »).

32.Theodor Adorno, Hanns Eisler, Musique de cinéma, Paris, L'Arche, 1972 [1947], p. 18. 